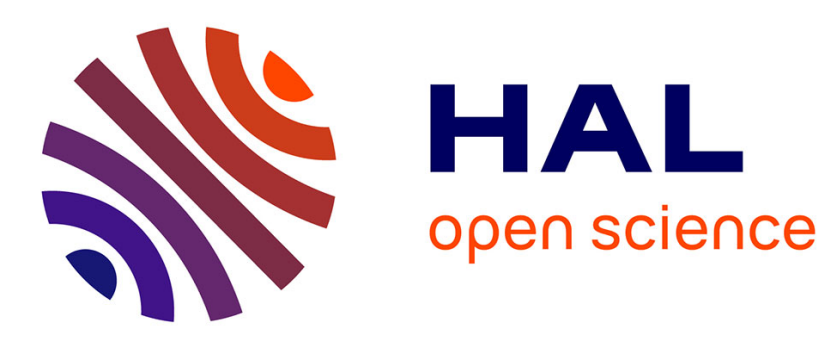

\title{
Le laboratoire des animal studies
}

Morgan Jouvenet

\section{To cite this version:}

Morgan Jouvenet. Le laboratoire des animal studies: Nature et culture dansles relations interspécifiques. Zilsel: science, technique, société, 2020, $\mathrm{N}^{\circ} 7$ (2), pp.161-178. 10.3917/zil.007.0161 . hal03082505

\section{HAL Id: hal-03082505 https://hal.science/hal-03082505}

Submitted on 18 Dec 2020

HAL is a multi-disciplinary open access archive for the deposit and dissemination of scientific research documents, whether they are published or not. The documents may come from teaching and research institutions in France or abroad, or from public or private research centers.
L'archive ouverte pluridisciplinaire HAL, est destinée au dépôt et à la diffusion de documents scientifiques de niveau recherche, publiés ou non, émanant des établissements d'enseignement et de recherche français ou étrangers, des laboratoires publics ou privés. 


\title{
Le laboratoire des animal studies. Nature et culture dans les relations interspécifiques
}

\author{
Morgan Jouvenet ${ }^{1}$ \\ «What a joy to know \\ even when we can't see you or hear you, \\ that you are around》 \\ W. H. Auden, «Address to the Beasts $»^{2}$.
}

\section{L'élan des animal studies et ses mobiles}

Si l'ambition de Zilsel, avec la publication d'un dossier consacré aux études sur les animaux, ou animal studies (AS), était de faire preuve d'originalité, l'échec était presque certain. L'intérêt pour les études menées en sciences humaines et sociales (SHS) sur les relations des hommes et des animaux, s'est encore confirmé ces derniers mois, notamment avec la publication de plusieurs dossiers de revues dédiés à cette question ${ }^{3}$. L'idée n'est donc pas d'initier ici un mouvement, ou bien de réactiver une thématique oubliée, mais plutôt de nourrir l'effervescence qui entoure aujourd'hui les AS, et plus particulièrement ce que l'on peut appeler l'étude des relations anthropozoologiques, c'est-à-dire les travaux « qui prennent pour objet à la fois les bumains et les animaux, en partant de situations qui les rassemblent $»^{4}$. Il s'agit de participer à un mouvement, et de contribuer au rayonnement - même momentané, car il n'y a pas de petit profit en la matière - de la réflexion sur ces relations anthropozoologiques et leurs inscriptions sociales.

Cette réflexion nous apparaît en effet très riche : outre les données empiriques inédites produites par ceux qui mènent l'enquête sur des terrains et des situations mettant aux prises hommes et animaux, ces travaux constituent une nouvelle mise à l'épreuve de dichotomies et concepts centraux pour les SHS, et pour les sciences studies qui nous intéressent tout particulièrement au sein de Zilsel. Comment les terrains animaliers incitent-ils à comprendre les rapprochements et différences que l'on peut faire entre les actions des humains et celles des non-humains, par exemple ? Ou à transformer les références savantes à des phénomènes « naturels » ou à des environnements « sauvages » ? L'idée était de permettre à des autrices et auteurs menant des enquêtes centrées sur les animaux de témoigner de la productivité intellectuelle de ces terrains, de montrer comment on pouvait s'y frotter, et ce qu'on pouvait tirer de ces frictions (c'est le but de cette rubrique éponyme).

L'intérêt de l'espèce humaine pour les autres espèces animales est l'une de ses marottes les plus anciennement documentées. Si les incertitudes sont grandes en la matière, les travaux des spécialistes des peintures rupestres laissent penser que cet intérêt a rapidement dépassé la seule logique alimentaire ${ }^{5}$. On peut dès lors considérer que les représentations des animaux, de leurs attitudes typiques et particularités individuelles, ont toujours nourri la compréhension par l'homme de la place qu'il occupe à côté d'eux sur notre planète - et donc que presque rien dans l'histoire humaine n'est moins neuf que cette prise en compte. En tant que cible, miroir, ou vecteur spirituel, l'animal est incontournable dans l'histoire des savoirs.

Cela n'empêche pas qu'il existe des séquences historiques et des configurations sociales et épistémiques dans lesquelles la question animale retentit avec davantage d'écho. L'essor des AS et l'audience qui leur est donnée

${ }^{1}$ CNRS, Laboratoire Printemps, morgan.jouvenet@uvsq.fr.

${ }^{2}$ In : Thank You, Fog: Last Poems (New York, Random House, 1974).

${ }^{3}$ Citons, parmi les plus récents : «Animals, Science and Technology: Multispecies Histories of Scientific and Sociotechnical KnowledgePractices» (HoST. Journal of History of Science and Technology, 2019), «La part animale du 19e siècle » (Revue d'histoire du $19^{e}$ siècle, $\mathrm{n}^{\circ}$ 54, 2017), «Les Sciences sociales et les animaux » (L'Année sociologique, vol. 66, 2016/2).

${ }^{4}$ Jérôme Michalon, Antoine Doré et Chloé Mondémé, «Une sociologie avec les animaux : faut-il changer de sociologie pour étudier les relations humains/animaux ? », SociologieS, 2016 (http:// sociologies.revues.org/5329), p. 7.

${ }^{5}$ Voir par exemple Jean Clottes, L'art des cavernes prébistoriques, Phaidon, Paris, 2008, et Pourquoi l'art prébistorique?, Paris, Gallimard Folio, 2011. 
depuis quelques années, au sein des SHS et au-delà, en témoignent. Les animaux sauvages, en particulier, sont les héros de plusieurs des essais les plus commentés de la vie intellectuelle récente. En France, Croire aux fauves, le livre d'une anthropologue transformée par sa rencontre accidentelle avec un ours, sur la presqu'île volcanique à l'est de la Russie ${ }^{6}$, a connu fin 2019 un succès proche de celui d'un philosophe dont la fréquentation des loups a conduit à proposer « une autre carte du vivant»7, en 2016. Dans un autre style, la rare panthère des neiges s'est récemment trouvée au cœur de la vie littéraire, via un livre de Sylvain Tesson fondé sur le récit d'une traque contemplative dans l'Himalaya (Prix Renaudot 2019). À l'étranger aussi, les expériences les plus personnelles de la vie sauvage se mêlent à la philosophie, à l'éthologie et d'autres sciences pour nourrir ce rayon d'essais à succès. L'une des plus saisissantes est la prise de conscience très concrète, par la philosophe Val Plumwood, que son corps « constitue pour d'autres de la nourriture », au moment où un crocodile l'arrache à son canoë. Après cela, le savoir " abstrait et désincarné » sur les relations hommeanimal apparaît bien désuet ${ }^{8}$. D'autres, artistes ou intellectuels, ont porté cette rupture avec une perception jugée trop abstraite de la vie animale à la pointe extrême de la radicalité (et de l'absurdité ?), à travers des expériences non accidentelles cette fois : ces tentatives pour « prendre congé du genre bumain » en se transformant en bouc (.. avec des prothèses de pattes et un estomac artificiel) $)^{9}$, ou bien pour en finir avec « le snobisme ontologique » en vivant six semaines comme un blaireau (puis comme un renard urbain et un cerf, pourchassé par un chien engagé pour l'occasion) ${ }^{10}$, ont été comprises comme des tentatives désespérées pour saisir l'altérité animale ${ }^{11}$, in vivo, en quelque sorte.

Ces rapprochements ne sont pas sans ambivalence. Parfois, ils servent à rappeler l'incommensurabilité des perspectives entre hommes et animaux, source de malentendus plus ou moins dangereux. C'est plutôt alors la grande naïveté et les limites cognitives des premiers - peut-être simplement « pas assęintelligents pour mesurer combien les animaux le sont $»^{12}-$ qui sont mises à l'index. Mais, d'autres fois, ils incitent plutôt à souligner les parallélismes entre «sociétés animales » et humaines - par exemple lorsque les premières se révèlent être des espaces dans lesquels l'individualité et la personnalité des animaux s'expriment sans les entraves habituellement associées aux traits typiques définissant les espèces dans le bestiaire des humains. C'est ce que montre par exemple Baptiste Morizot dans Les diplomates, au sujet des loups, à partir d'observations réalisées sur des meutes sauvages ${ }^{13}$. Comme l'écrit Kate Soper, "si nous nous sommes tournés vers les animaux pour tenter de transcender la "solitude" on "l'aliénation" de notre statut culturel, nous les avons aussi constamment utilisés pour maintenir en place la barrière culture-nature »: autrement dit, parler des animaux nous sert aussi bien à 《contrôler » qu'à « brouiller la frontière entre l'homme et la nature »"

Le savoir scientifique sur les animaux est incontournable dans ces réflexions. Les exemples mentionnés à l'instant en témoignent: qu'elles cherchent à dépasser ou à articuler ce savoir à des expériences plus incarnées des relations avec eux, philosophes, anthropologues, et sociologues nourrissent un dialogue renouvelé entre les SHS et la biologie animale ou l'éthologie. L'intérêt de Zilsel pour les AS s'explique aussi

\footnotetext{
${ }^{6}$ Nastassja Martin, Croire aux fauves, Paris, Verticales, 2019.

${ }^{7}$ Baptiste Morizot, Les diplomates. Cohabiter avec les loups sur une autre carte du vivant, Paris, Éditions Wildproject, 2016.

${ }^{8}$ Val Plumwood, The Eye of the Crocodile, Canberra, Australian National University Press, 2012. La revue Terrestres en a traduit récemment un extrait : https://www.terrestres.org/2019/01/16/loeil-du-crocodile/. Voir aussi «Penser avec le corps. Comment une panthère a transformé ma thèse ", où Jean Estebanez raconte sa rencontre avec une panthère, dans un zoo (https://hal.archives-ouvertes.fr/hal-01659616).

9 Thomas Thwaites, GoatMan: How I Took a Holiday from Being Human, Princeton, Princeton Architectural Press, 2016.

${ }^{10}$ Charles Foster, Being a Beast, Londres, Metropolitan, 2016.

${ }^{11}$ Elles ont dès lors été mises en regard du texte classique du philosophe Thomas Nagel, «What Is It Like to Be a Bat?» (The Philosophical Review, vol. 83, n 4, p. 435-450, 1974). Voir par exemple Joshua Rothman, «The Metamorphosis. What is it like to be an animal? ", The New Yorker, vol. XCII, n 16, 30 mai 2016 (https://www.newyorker.com/magazine/2016/05/30/goatman-and-being-a-beast).

${ }^{12}$ Frans de Waal, Are We Smart Enough to Know How Smart Animals Are?, Londres, Granta, 2016

${ }^{13}$ Baptiste Morizot, Les diplomates. Cohabiter avec les loups sur une autre carte du vivant, op. cit., p. 146-168. ("Un laboratoire nommé Yellowstone »).

${ }^{14}$ Kate Soper, What is Nature? Culture, Politics and the Non-Human, Cambridge, Blackwell, 1995, p. 87. Elle rappelle, à la suite de Raymond Williams (Problems in Materialism and Culture, Londres, Verso, 1980, p. 68), que le mot «nature» est "l'un des plus complexe du langage » : c'est une « formation culturelle » dont on peut analyser l'histoire et l'ambivalence - sans pour autant considérer qu'elle ne renvoie qu'à des éléments discursifs (What is Nature?, p. 249). Rappelant également les ambiguités du terme (en renvoyant cette fois à Carolyn Merchant, The Death of Nature: Women, Ecology), and the Scientific Revolution, New York, Harper Colins, 1990), Virginie Marris a aussi défendu récemment la pertinence conceptuelle et politique de l'idée de la nature « comme cette part du monde que nous n'avons pas créée » (La part sauvage du monde. Penser la nature dans l'Anthropocène, Paris, Seuil, 2018, p. 21-28).
} 
par cette reconfiguration des rapports entre disciplines et spécialités. Celles-ci évoluent suivant des logiques diverses : l'apparition de nouveaux instruments, ou la miniaturisation de plus anciens, par exemple, est un facteur qui a sensiblement accru la connaissance scientifique sur les comportements de nombreuses espèces et individus. On peut penser aux études sur les migrations ${ }^{15}$, mais aussi à celles qui visent les modes de communication animale, ou encore leur sensibilité (voire leurs "émotions»). De même, la nouvelle puissance des techniques de manipulation des données numériques (relevant des "big data») a considérablement transformé les conceptions de la biodiversité ${ }^{16}$. En la matière, les spécialistes évoquent "une explosion des moyens de description du vivant [qui] a bouleversé nos moyens de se représenter la connaissance et la protection de la nature $\aleph^{17}$. Il est toutefois difficile, surtout au regard de plusieurs décennies de science studies, d'imaginer que ces évolutions - spécialisées ou interdisciplinaires - ne puissent être rapportées qu'à une inéluctable évolution instrumentale, isolée du contexte social dans lequel vivent et travaillent les scientifiques.

\section{Homme-animal : nouvelles perspectives}

Comme le souligne Dominique Guillo, une grande partie des études sur les animaux a, ces dernières décennies, été largement influencée par la place prise par les sciences cognitives et les neurosciences dans l'écologie de la recherche (entraînant notamment « un déplacement du noyau conceptuel des parties les plus théoriques de l'éthologie $»^{18}$ ). Telles qu'elles peuvent être appliquées en laboratoire aux animaux, ces spécialités transforment en effet notre connaissance d'eux, et interrogent nos différences vis-à-vis de certaines espèces sur des bases nouvelles. Sur ce plan, la réflexion sur l'intelligence, ou sur la conscience animale, apparaissent symétriques de celle sur l'intelligence artificielle ${ }^{19}$, dans le sens où elle est également révélatrice d'un modèle de l'acteur humain. Les relations «entre l'bumanité et l'animalité (et notamment les autres primates)» figurent ici comme cas ultime pour déterminer, moins la validité de «la division bumanité-nature » en elle-même, que celle de sa conceptualisation comme dichotomie catégorielle ou variance sur une même échelle ${ }^{20}$. Ses résultats peuvent aussi conduire à réexaminer l'articulation entre l'agentivité des humains et celle des animaux - leur "part active dans les dynamiques sociales »"1 - au fil des récits produits par les SHS. Cette question a joué un rôle central dans les querelles théoriques des science studies (à partir des propositions de Bruno Latour sur les «non-humains", notamment ${ }^{22}$ ). Sa pertinence pour les SHS est toutefois bien plus générale. Pour s'en rappeler, on peut par exemple évoquer la célèbre distinction que faisait Marx entre « le travail » d'une abeille et celui d'un architecte, et aux nombreux commentaires auxquels elle a donné lieu.

Les situations de travail associant hommes et bêtes apparaissent comme une source de questionnement particulièrement riche, dans la mesure où elles rapprochent les êtres sur un plan affectif autant qu'elles nécessitent une coordination des intelligences. La participation des animaux aux activités humaines est parfois involontaire : ainsi l'enrôlement des éléphants de mer « augmentés » (i.e. affublés de capteurs) dans l'étude des températures et de la salinité de la banquise antarctique, ou de l'état de la mer ${ }^{23}$, ou celui des albatros dans la détection de la pêche illégale ${ }^{24}$, ne nécessite de leur part aucun apprentissage particulier, et

\footnotetext{
${ }^{15}$ James Cheshire et Oliver Uberti, Atlas de la vie sauvage. Comment les nouvelles technologies permettent de suivre et de comprendre les déplacements des animaux, Paris, Les Arènes, 2017.

16 Sur les limites et risques de la perception de «la nature comme base de données», celles d'« un double mouvement d'hypersurveillance et de virtualisation de la nature ", voir Virginie Marris, La part sauvage du monde, op. cit., p. 169-187, 201.

17 Vincent Devictor, Nature en crise. Penser la biodiversité, Paris, Seuil, 2015, p. 248-249.

${ }^{18}$ Dominique Guillo, Les fondements oubliés de la culture. Une approche écologique, Paris, Seuil, 2019, p. 40-42.

${ }^{19}$ Sur l'IA, voir le dossier «Frictions» de Zilsel n 5 coordonné par Olessia Kirtchik («Les STS et l'intelligence artificielle : une rencontre manquée ?»).

${ }^{20}$ Kate Soper, What is Nature?, op. cit., p. 41-42.

${ }^{21}$ Jérôme Michalon, "La cause animale et les sciences sociales : influences, dévoilements et appariements », in Fabien Carrié et Christophe Traïni (dir.), S'engager pour les animaux, Paris, Presses universitaires de France, 2019, p. 89-101. Cette insistance sur l'agentivité animale distingue les nouvelles recherches en AS de " tout un pan de recherches sociologiques [qui] a abordé les animaux en tant que supports matériels et symboliques des sociétés humaines [...], en tant que révélateurs du fonctionnement de ces dernières [...], ou encore en tant que vecteurs du pouvoir social et politique des hommes » (Jérôme Michalon, Antoine Doré et Chloé Mondémé, «Une sociologie avec les animaux », art. cit., ils soulignent).

22 Pour une synthèse de ces propositions, voir Gerard De Vries, Bruno Latour. Une introduction, Paris, La Découverte, 2018, p. 71-104.

${ }^{23}$ Cf. CNES Mag nº 81 (« Le vent et les vagues. Un couple énigmatique »), p. 32.

${ }^{24}$ Henri Weimerskirch et al., «Ocean sentinel albatrosses locate illegal vessels and provide the first estimate of the extent of nondeclared fishing», Proceedings of the National Academy of Sciences, 201915499 ; DOI:
} 
des interactions peu développées avec les humains. C’est déjà moins le cas pour les animaux d'élevage : les travaux de Jocelyne Porcher notamment, ont montré les liens forts qui peuvent les unir à leurs éleveurs ${ }^{25}$. Il en va encore autrement des éléphants d'Asie, dont l'intégration à des collectifs de travail et la coopération avec un cornac reposent sur un « investissement» que des enquêtes sociologiques ont récemment détaillé26. Dans ce cas, comme dans celui des animaux militarisés (des chevaux transporteurs de troupes aux bélougas porteurs de mines sous-marines), la question du «bien-être animal» et des attentions à accorder à un individu particulier ne peut manquer d'être articulée à celle des bénéfices engendrés pour l'homme - ou pour d'autres espèces.

C'est l'occasion de rappeler aussi que le détail des comptes rendus d'enquêtes anthropozoologiques, les qualifications qu'ils mettent en œuvre, et les représentations qu'ils promeuvent, ont des effets pratiques, et peuvent fonder des attitudes et des politiques très différentes en direction des animaux. Comme l'écrit $\mathrm{K}$. Soper, "nos discours sur la nature sont constitutifs d'une série de conceptions et de représentations à travers lesquelles nos politiques de l'environnement sont nécessairement médiatisées ${ }^{27}$. Les politiques de conservation sont bien sûr parmi les plus évidemment sensibles à ces discours, en tant qu'« entreprises imprégnées de la pensée de la nature et mêlant la science, la politique et cette expérience pratique de la vie marquée aussi bien par le rêve de la maîtrise que par celui du naturalisme $»^{28}$. C'est l'une des leçons du texte présenté dans ce dossier par Isabelle Arpin. Par exemple, la politique déployée dans un parc naturel peut varier en fonction du statut que l'on accorde à telle ou telle espèce, et aux individus au sein de cette espèce. Charis Thompson a ainsi évoqué différentes représentations des éléphants (sauvages) d'Afrique comme des « métonymies» de politiques de la nature, pouvant être fondées, soit sur l'idée que les éléphants vivant dans un parc constituent une " espèce clé de voûte » sur laquelle l'homme peut intervenir à sa guise pour " gérer » la biodiversité, soit sur l'idée que « chaque éléphant a des droits intrinsèques, découlant de sa vie sociale et mentale complexe $»^{29}$.

Des images d'animaux se trouvent aujourd'hui massivement diffusées, sous des formats très divers, de la série documentaire vedette transnationale sur la vie sauvage (e.g. « Notre planète » $\left.{ }^{30}\right)$ aux vidéos amateurs de chiens, de chats ou de pandas roux qui se renouvellent chaque jour sur Internet. Comme l'écrit J. Lorimer à la suite de son enquête sur les éléphants du Sri Lanka, nos relations avec les bêtes se nourrissent aussi de cette "écologie médiatisée, à travers laquelle les publics occidentaux sont beaucoup plus susceptibles de rencontrer les êtres charismatiques qui les préoccupent tant dans la presse écrite, en ligne ou à la télévision, que de les rencontrer en chair et en os", dans leur environnement naturel (ou même dans un zoO ${ }^{31}$ ) : autrement dit, ces images de la vie animale

10.1073/pnas.1915499117, 2020. Cf. aussi « Des albatros pour repérer les pêcheurs illégaux dans les mers australes », Le Monde, 28 janvier 2020.

${ }^{25}$ Voir Jocelyne Porcher, Éleveurs et animaux, réinventer le lien, Paris, Presses universitaires de France, 2002 ; id., La Mort n'est pas notre métier, La Tour d'Aigues, Éditions de l'Aube, 2003 ; et Jocelyne Porcher et Vinciane Despret, Étre bête, Arles, Éditions Actes Sud, 2007.

${ }^{26}$ Nicolas Lainé, « Coopérer avec les éléphants dans le Nord-Est indien », Sociologie du Travail, vol. 60, n² 2, 2018, URL : http://journals.openedition.org/sdt/1953. Voir aussi, Jacob Shell, Giants of the Monsoon Forest: Living and Working with Elephants, New York \& Londres, Norton \& Company, 2019.

${ }^{27}$ Kate Soper, What is Nature?, op. cit., p., p. 249.

${ }^{28}$ Jamie Lorimer, Wildlife in the Anthropocene: Conservation After Nature, Mineapolis, Londres, University of Minnesota Press, 2015, p. 5. Pour lui, la question de la conservation des espèces relève d'« une biopolitique et d'un biopouvoir » (p. 12 sq., 58-59). Elle est compliquée, dans le cas des éléphants du Sri Lanka, par leur « bybridité » : «ils sont trop sociaux et malins pour être des objets, trop étranges pour être des humains, trop captifs pour être sawvages, mais aussi trop sawvages pour être domestiqués » (p. 20). Sur cette question, voir aussi Fernando Vidal et Nélia Dias (dir.), Endangerment, Biodiversity and Culture, Londres \& New York, Routledge, 2015.

${ }^{29}$ Charis Thompson, «When Elephants Stand for Competing Philosophies of Nature: Amboseli National Park, Kenya », in John Law et Annemarie Mol (eds.), Complexities: Social Studies of Knowledge Practices, Durham \& Londres, Duke University Press, 2002, p. 167-171. Sur l'histoire et la politique des parcs nationaux, voir Bernhard Gissibl, Sabine Höhler et Patrick Kupper (dir.), Civilizing Nature: National Parks in Global Historical Perspective, New York \& Oxford, Berghahn Books, 2012.

30 https://www.ourplanet.com/fr/

${ }^{31}$ Lorimer rappelle aussi combien les zoos, qui « se présentent de plus en plus comme des institutions vitales pour la conservation » de certaines espèces, jouent un rôle important dans l'« économie affective de la vie sauvage » : «ils collectent des fonds, sensibilisent et éduquent le public, mènent des recherches et accueillent des populations d'espèces rares » (Jamie Lorimer, Wildlife in the Anthropocene: Conservation After Nature, Mineapolis, London, University of Minnesota Press, 2015, p. 145-146). Cette évolution a cependant fait l'objet de nombreuses critiques, et revivifié la littérature sur le sujet. Voir par exemple Irus Braverman, Zooland: The Institution of Captivity, Stanford, Stanford University Press, 2013, et Thomas French, Zoo Story: Life in the Garden of Captives, New York, Hyperion, 2010. 
"façonnent la façon dont les gens se sentent, pensent et agissent à l'égard du monde non humain »32. Et elles sont de plus en plus explicitement conçues dans ce but. Depuis quelques années, en France, l'association L214 s'est par exemple fait une spécialité de faire voir à un maximum de personnes les traitements les plus violents infligés à des bêtes, afin de les sensibiliser à la question de la souffrance animale (et les mobiliser contre certains acteurs de la filière agro-alimentaire). D'autres vidéos, moins explicitement politiques, travaillent à l'inverse à documenter la proximité des hommes et des bêtes en témoignant de l'intensité de leurs relations (de leur « complicité »). L’enregistrement de la « dernière entrevue» d'un chimpanzé mourant, prostré et refusant toute nourriture, avec une vieille connaissance humaine, en est une bonne illustration, qui a fait l'objet d'innombrables reprises médiatiques ${ }^{33}$.

Une autre vidéo a connu un succès similaire, quelques mois plus tard : dans un style différent, elle montrait un orang-outang manifestant une certaine agitation face aux dégâts causés par l'activité d'un bulldozer dans son habitat «naturel $»^{34}$. Dans ce cas, on voit bien que la réaction du primate (et l'éventuelle adhésion de ses spectateurs humains à sa révolte) prend sens dans le contexte d'une réflexion critique sur l'impact des activités humaines sur l'environnement. La démographie et les comportements animaux constituent en effet des objets de réflexion particulièrement porteurs pour mesurer cet impact, et pour sensibiliser le public à leurs conséquences et à leurs conditions sociales de possibilité - que l'on annonce « la fin de la nature $»^{35}$ en insistant sur l'omniprésence nocive de l'empreinte de l'homme, ou bien que l'on considère au contraire que l'empiétement des activités humaines sur «la part sawvage du monde » ne fait qu'en exacerber sa valeur esthétique et morale ${ }^{36}$. En ce sens, il faut rapprocher aussi l'intérêt pour les animaux - pour leurs difficultés, et pour la dépendance de leur existence vis-à-vis de ces activités, en tant que "cohabitants " $^{37}-$ de l'interrogation plus générale qui porte aujourd'hui sur le rapport de l'homme à la nature et à ses composantes « à l'ère de l'anthropocène ».

La leçon que l'on tire, dans ce cadre, des travaux d'histoire environnementale montrant combien les éléments naturels - et parmi eux les animaux ${ }^{38}$ - ont pu jouer un rôle dans l'histoire humaine ${ }^{39}$, indique actuellement plutôt que le retour de bâton d'une nature maltraitée sera très coûteux. Les effets de cette maltraitance sont désormais bien connus : déforestation, pollution massive des sols et des océans, réchauffement climatique accéléré... Autant de problèmes auxquels l'humanité a et aura du mal à faire face, du moins pour ce qui concerne ses populations les moins favorisées ${ }^{40}$. Pour les autres espèces, plus ou moins emblématiques, les perspectives sont plus noires encore, et ont conduit de nombreux spécialistes à annoncer que nous vivons aujourd'hui les prémisses d'une $6^{\mathrm{e}}$ extinction de masse ${ }^{41}$. En tout état de cause, la biodiversité de nombreuses régions du globe est sérieusement menacée, et devient l'objet de débats scientifiques et politiques aujourd'hui très visibles ${ }^{42}$.

\footnotetext{
32 Jamie Lorimer, Wildlife in the Anthropocene, op. cit., p. 119.

33 Voir par exemple «Watch The Moment A Dying Chimpanzee Recognizes An Old Friend» (https:/ / www.npr.org/sections/13.7/2017/10/24/559837354/watch-the-moment-a-dying-chimpanzee-recognizesan-old-friend? $\mathrm{t}=1579770690068)$. Cette femelle chimpanzé est bien connue des primatologues, car elle est l'une des héroïnes (à la personnalité particulièrement affirmée) de l'un des livres de Frans de Waal (Chimpanzeee Politics: Power and Sex among Apes, New York, Harper \& Row, 1982).

34 Voir par exemple «Orangutan Filmed Trying to Fight off Digger Destroying its Jungle Home », https://www.independent.co.uk/news/world/asia/orangutan-defends-jungle-home-video-digger-ape-borneoindonesia-deforestation-a8387836.html).

35 Bill MacKibben, The End of Nature: Humanity, Climate Change and the Natural World, New York, Random House, 1989.

36 Virginie Marris, La part sauvage du monde, op. cit.

${ }^{37}$ Baptiste Morizot, «Le devenir du sauvage à l'Anthropocène », in Rémi Beau et Catherine Larrère (dir.), Penser l'Anthropocène, Paris, Presses de la Fondation nationale des sciences politiques, 2018, p. 249-264.

38 Par exemple le moustique, décrit comme l'« agent ultime du changement historique », notre « apex predator» et « destructeur de monde », par Timothy C. Winegard (The Mosquito: A Human History of Our Deadliest Predator, New York, Dutton, 2019).

${ }^{39}$ Pour n'en citer qu'un exemple: Kyle Harper, Comment l'Empire romain s'est effondré. Le climat, les maladies et la chute de Rome, Paris, La Découverte, 2019.

40 Andreas Malm, The Progress of this Storm: Nature and Society in a Warming World, Londres, Verso, 2018.

${ }^{41}$ Elisabeth Kolbert, La sixième extinction. Comment l'homme détruit la vie., Paris, Vuibert, 2015.

42 Vincent Devictor, Nature en crise, op. cit. Il montre notamment combien cette question appelle une "nouvelle éthique environnementale ».
} 


\section{Les textes du dossier}

On comprend que les études sur les animaux, et sur les relations qu'ils entretiennent avec les hommes, en tant que foyer de définition socio-historique du rapport à la nature ${ }^{43}$, prennent une importance nouvelle dans ce contexte. Les enjeux sociaux et politiques que je viens d'esquisser expliquent du reste que les débats soient particulièrement vifs au sein des AS. Le texte de J. Porcher inclus dans le présent dossier l'atteste clairement, d'une part en mettant en évidence le lien entre certaines conceptions de «la défense des animaux» et certains courants de ces AS (c'est en ce sens que Jérôme Michalon, Antoine Doré et Chloé Mondémé ont pu évoquer les AS comme «une sociologie pour les animaux» $\rangle^{44}$ ), et d'autre part en fondant un choix politique (celui du refus de la viande artificielle) sur une conceptualisation originale du travail animal.

Avec ce dossier, notre volonté à Zilsel n'était pas cependant de défendre une thèse ou une approche en particulier. Il s'agissait plutôt de présenter différentes entrées pour l'enquête sur l'inscription sociale des animaux, ainsi que les points de vue et options théoriques qui peuvent s'y rattacher. Dans cette optique, il nous a paru pertinent, notamment, de donner à lire à la fois les propositions théoriques et le programme empirique promus par D. Guillo pour renouveler les AS (et plus généralement, les liens entre SHS et éthologie), qu'il présente dans son article, et leur commentaire et critique par J. Michalon et A. Doré.

Comme il l'a aussi écrit dans son dernier ouvrage, Les fondements oubliés de la culture. Une approche écologique, les propositions de D. Guillo visent d'abord à déconstruire «le mur d'indifférence, d'ignorance, ou de rejet » qui entrave un rapprochement qu'il estime fécond, en tant que sociologue, entre les SHS et l'éthologie ${ }^{45}$. Critiquant la thèse du " Grand Partage », il montre que ce mur n'est pas né avec les premières, mais qu'il a été constitué au cours de leur histoire, et de manière d'autant plus dommageable aujourd'hui que les secondes ont récemment développé d'importantes recherches sur les "phénomènes socioculturels » observables dans le monde animal. Il s'attache pour cela dans son article à pointer les «flottements et impasses» qui caractérisent la "perspective très bumaine » sur ce monde qu'ont développé des auteurs pourtant ostensiblement attachés à la prise en compte des non-humains dans leurs enquêtes. À travers ses propres enquêtes, il indique aussi combien il est important d'accorder davantage de place à des situations d'interaction entre hommes et animaux. Et, pour s'engager dans les enquêtes qu'il prône, mieux vaut oublier les vidéos à succès sur les primates comme celles que j'ai mentionnées plus haut : plutôt que de se concentrer sur les effets destructeurs des activités humaines, D. Guillo incite à s'intéresser à la productivité culturelle d'interactions a priori moins spectaculaires entre humains et animaux - entre des chiens d'aveugles et leurs maitres, par exemple, ou entre des macaques et des touristes. Les images qui révèlent la familiarité de certains primates avec certains hommes, aussi émouvantes qu'elles puissent être, présenteraient ainsi le risque de nous induire en erreur, en nous faisant oublier d'autres fondements des cultures. Et, au lieu de chercher des ressemblances, voire une «identité partagée » au principe des interactions, D. Guillo propose d'analyser ce que les relations « concrètes et situées » entre espèces font faire aux unes et aux autres.

L'ambition de ce programme appelait un commentaire de la part de spécialistes des AS, et J. Michalon et A. Doré en proposent une lecture serrée. Pour eux, Les fondements oubliés de la culture offre d'abord une présentation éclairante des questions complexes qui constellent ce domaine de recherche, et un point de vue érudit sur les avancées des sciences du comportement animal. Outre son approche non déterministe de la biologie et des sciences du comportement, ils saluent le souci de l'enquête multi-échelles qui caractérise sa démarche, qui détonne dans un "paysage intellectuel chargé de généralisations excessives, d'enjeux normatifs, et sowvent pawvre empiriquement». Si certains apports leur semblent décisifs (l'identification du "biais identitaire », notamment), D. Guillo a toutefois négligé, selon eux, d'autres aspects de ces questions : le manque de référence à l'anthropologie des techniques, par exemple, produit une «vision de la culture » qui leur parait malheureusement «déponillée des artefacts techniques et institutionnels». Ils voient aussi un manque de réalisme sociologique dans les regrets que D. Guillo émet au sujet du déficit d'interdisciplinarité des études sur les animaux. C'est en ce sens que ses propositions leur apparaissent trop exclusivement épistémologiques. De manière surprenante, ils trouvent des proximités entre l'approche de D. Guillo et celle de B. Latour et Michel Callon, et se demandent même si on ne pourrait pas assimiler son projet à une « modernisation de Latour».

\footnotetext{
${ }^{43}$ Jérôme Lamy et Romain Roy, «Introduction. Anthropologie historique de la nature : essai de cadrage et tentative de définition ", in Jérôme Lamy et Romain Roy (dir.), Pour une anthropologie historique de la nature, Rennes, Presses universitaires de Rennes, 2019, p. 18.

${ }^{44}$ Jérôme Michalon, Antoine Doré et Chloé Mondémé, "Une sociologie avec les animaux », art. cit., p. 8-10 (je souligne). Sur ce point, voir aussi Jérôme Michalon, « La cause animale et les sciences sociales : influences, dévoilements et appariements », in Fabien Carrié et Christophe Traïni (dir.), S'engager pour les animaux, op. cit., p. 89-101.

${ }^{45}$ Dominique Guillo, Les fondements oubliés de la culture, op. cit., p. 29, 15.
} 
Malgré cela, le projet de D. Guillo ne leur semble pas compatible avec tous les paradigmes théoriques, du fait de sa focalisation (qu'ils estiment excessive) sur les interactions, et ils concluent leur texte en exprimant leur scepticisme vis-à-vis de ce qu'ils perçoivent comme « la dimension totalisante » de ce projet.

C'est une réserve importante, qui a fait réagir D. Guillo. À notre invitation, les trois auteurs ont prolongé le dialogue, dans deux textes destinés à répondre aux critiques qui leur sont adressées. D. Guillo estime que celles de A. Doré et J. Michalon témoignent justement de la méconnaissance des acteurs de SHS pour l'éthologie, et il regrette les termes généraux avec lesquels ils ont évalué ses propositions et situé son livre. Sa réponse est aussi pour lui l'occasion d'affirmer le caractère complémentaire de l'analyse écologique qu'il promeut. Ainsi, la mise au jour du «biais identitaire» vise moins à «contester la puissante assise qu'offre l'identité partagée au déploiement de la culture » qu'à attirer l'attention sur d'autres phénomènes (" d'ajustement de différences ») dans l'émergence des cultures. Assumant leur volonté d'étendre le débat sur les relations humains-animaux à un lectorat plus large que ses seuls animateurs, S. Michalon et A. Doré rappellent de leur côté dans leur réponse l'intérêt qu'il peut y avoir à mettre un ouvrage en regard de travaux proches, "antérieurs ou contemporains", et à le situer dans un espace académique large (et dans l'histoire intellectuelle qui l'a modelé). L'ampleur du programme de D. Guillo justifiait selon eux d'entreprendre cette mise à l'épreuve, quitte à en déplacer un peu le cadre de lecture. Au-delà des oppositions exprimées ici, et des convictions qu'elles ont affermies de part et d'autre des claviers, la lecture de ce «dossier dans le dossier», consacré au livre Les fondements oubliés de la culture, offre en tout cas une entrée particulièrement riche pour penser l'ancrage des animal studies dans les sciences sociales.

Deux articles du dossier reposent sur des analyses des relations entre hommes et animaux qui se déroulent dans des configurations sociales très différentes : si celui de Chloé Mondémé oppose les conceptions de l'intentionnalité et de l'esprit des animaux forgées au sein des sciences cognitives à celles qui sont mises en œuvre, pratiquement, dans des interactions ordinaires, celui d'Isabelle Arpin part d'une affaire de politique environnementale centrée sur des bouquetins, pour interroger les représentations que ses acteurs déploient pour penser le sauvage. La première part d'une problématisation scientifique douteuse, la seconde conclut son analyse sur l'utilité pratique des savoirs scientifiques.

L'affaire analysée par I. Arpin démarre avec "un événement inattendu», une surprise sanitaire et scientifique : celui de la transmission d'une bactérie que l'on pensait impossible, des bouquetins aux humains, via des vaches. L'autrice nous présente le problème posé par les bouquetins du massif du Bargy (Haute-Savoie), et propose une réflexion sur les frontières et les dualismes qui sont déployés par les acteurs de cette affaire, et dans les humanités environnementales : humain/animal, sauvage/domestique, nature/culture. À partir des données empiriques qu'elle a recueillies lors d'une observation participante « experte », elle montre comment la force donnée à ces distinctions peut déterminer les actions mises en œuvre sur le terrain, qu'il s'agisse de protéger les animaux ou de traiter la maladie. Pour les bouquetins, c'est même une question de vie ou de mort. En l'espèce, la brucellose est une maladie révélatrice des liens entre les équipements conceptuels de diverses catégories d'acteurs et les politiques de conservation qu'ils sont susceptibles de promouvoir dans un espace carrefour, modelé par des activités économiques et touristiques, et abritant "une communauté multispécifique, incluant (entre autres) des bumains, des vaches, des bouquetins et des brucelles». À l'issue d'une réflexion tournée vers l'action, orientée par la question " que faire ? ", I. Arpin assume une conclusion plutôt engagée - dans le sens où elle affirme la supériorité de la démarche et du savoir scientifique. Sur ce terrain, et face aux questions éprouvant cette "communauté multispécifique », ce sont en effet les outils et savoirs scientifiques qui se sont révélés les plus efficaces, en permettant «de discriminer utilement les animaux [...], d'envisager et d'explorer de nouveaux scénarios, de décider en meilleure connaissance de cause comment intervenir, et d'informer et d'éclairer le débat public.»

Dans un autre style, l'article de C. Mondémé s'appuie également sur un important travail de terrain pour questionner des routines conceptuelles - celles qui définissent les contours de «l'intentionnalité animale », dans plusieurs spécialités scientifiques. L'originalité de son approche ("pragmatiste et inspirée de l'ethnométhodologie ») réside dans le fait de déplacer le questionnement «d'un vieux débat scolastique, constamment rejoué dans la sphère scientifique contemporaine », opposant nature et culture, l'instinct et le raisonnement, à la sphère pratique des "individus et groupes qui vivent ordinairement au contact d'animaux, de manière quotidienne (propriétaires, vétérinaires, entraineurs) ou plus ponctuelle (promeneurs dans les parcs, visiteurs de zoo, etc.) ». Avant de montrer le bénéfice heuristique de ce déplacement, C. Mondémé revient sur les apories du traitement de l'intentionnalité animale dans les sciences cognitives. Pour elle, le problème se situe à la fois dans la circularité du raisonnement qui y est mis en œuvre pour saisir l'existence d'une «théorie de l'esprit» chez l'animal - autrement dit leur « capacité d'imputer des états mentaux à eux-mêmes ou à autrui» - et dans sa « retraduction empirique dans des protocoles expérimentaux », qui reposent sur des généralisations excessives et une essentialisation fautive. C. Mondémé 
invite à déplacer la question sur un terrain plus commun : celui circonscrit par des interactions relativement banales avec des animaux, au cours desquelles leur intentionnalité n'apparaît plus comme un objet théorique fuyant, mais comme une ressource pratique. De son point de vue, cette "respécification ethnométhodologique » rehausse la contribution des SHS, face au défi de la saisie de l'intentionnalité animale.

Le dernier texte du dossier, celui de Jocelyne Porcher, déploie une perspective encore différente, mobilisant des analyses sociologiques tranchées pour affirmer les vertus d'une politique de la viande animale. L'autrice déploie d'abord une critique des promesses technologiques et industrielles de nouveaux acteurs de l'industrie alimentaire, producteurs plus ou moins crédibles d'une «clean meat» de masse, offrant aux consommateurs une alternative à la viande issue de l'élevage et de l'abattage d'animaux (sous la forme d'un produit issu de la culture de cellules animales). L'amplitude de ces promesses est considérable, puisqu'il est question de « sauver la planète et les animaux», en s'appuyant sur de nouvelles techniques de production " propres » (in vitro). J. Porcher montre que la promotion de ce «monde meilleur» et de sa politique désincarnée s'appuie sur des représentations forgées par des biologistes, des chercheurs en SHS, et par les militants pro-animaux. Elle attire l'attention sur les ressorts sociologiques de cet assemblage aux intérêts partagés, et revient en particulier sur le rôle joué par les spécialistes des AS, qui ont déplacé le centre de gravité des enquêtes sur les animaux de l'analyse des relations entre humains et animaux (Human-Animal Studies) vers la critique de leur exploitation et domination, avant de « radicaliser une position zoocentrée ». Ce déplacement thématique, destiné à revaloriser le rôle (et le point de vue) des animaux dans les sociétés humaines, a achevé de faire disparaitre «la centralité du travail dans nos relations aux animaux ». Or, J. Porcher voit précisément dans cette forme de vie une composante essentielle d'une coexistence saine et morale avec les bêtes. Pour finir, elle dépeint la société dont la «clean meat» est le vecteur, légitimant une nouvelle production alimentaire "à deux vitesses », entre une filière d'agriculture cellulaire archi-dominante, et " quelques troupeaux survivant dans des territoires préservés» et donnant de la viande et des produits laitiers étiquetés pour la consommation de luxe. C'est pour conjurer cette vision qu'elle estime dangereuse que J. Porcher mise sur une sociologie du travail animal, en rupture avec ces représentations de «l'animal-viande » et de «l'animal victime », qui dominent aujourd'hui les AS. 